\title{
On the choice of contact parameters for the forced response calculation of a bladed disk with underplatform dampers
}

\section{Original article}

Article history:

Accepted: 10 April 2017

Published: 20 June 2017

This paper is the updated version of a paper originally presented at the 1 st Global Power and Propulsion Forum, GPPF 2017, in Zurich, Jan 16-18 2017

\section{Check for updates}

*Correspondence:

CG: chiara.gastaldiapolito.it

Peer review:

Single blind

\section{Copyright:}

(c) 2017 Gastaldi et al. @ This is an open access article distributed under the Creative Commons Attribution Non Commercial License (CC BY-NC 4.0), which permits unrestricted use, distribution, and reproduction in any medium for noncommercial purposes only, provided the original work is properly cited and its authors credited.

\section{Keywords:}

bladed disk; contact stiffness; friction coefficient; friction damping; harmonic balance method; underplatform damper

\section{Citation:}

Gastaldi C., Grossi E., and Berruti T. M. (2017). On the choice of contact parameters for the forced response calculation of a bladed disk with underplatform dampers. Journal of the Global Power and Propulsion Society. 1: 1-15.

https://doi.org/10.22261/5D19RH
Chiara Gastaldi ${ }^{1, *}$, Emanuele Grossi ${ }^{2}$, Teresa M. Berruti ${ }^{1}$

\author{
${ }^{1}$ Politecnico di Torino, Corso Duca degli Abruzzi 24, DIMEAS, 10129 \\ Torino, Italy \\ ${ }^{2}$ Department of Mechanical and Industrial Engineering, University of \\ Illinois at Chicago, Chicago, Illinois, 60607, USA
}

\begin{abstract}
Underplatform dampers (UPDs) are still in use to reduce the vibration amplitude of turbine blades and to shift the position of resonant frequencies. The dynamics of blades with UPDs is nonlinear and the analysis is challenging from both the experimental and the numerical point of view. A key point in obtaining a predictive numerical tool is the choice of the correct contact parameters (contact stiffness and friction coefficient) that are required as input to the contact model. The paper presents different approaches to choose these parameters: the contact stiffness in normal and tangential direction are both calculated and measured. The calculation is based on the analytical models in literature, the measurements are carried out on a dedicated test rig. The friction coefficient is also measured. Test results of the forced response of the same bladed disk with UPDs are available for each blade, they come from an experimental campaign under controlled excitation and centrifugal force. The forced response of the bladed disk is not used as a mean to tune the contact parameters, but rather as a validation tool: the effect of the different choices of contact parameters in the code is highlighted by the comparison of the calculated and experimental forced response of the bladed disk.
\end{abstract}

\section{Introduction}

The study of the dynamic behavior of turbine bladed disks vibration is still a topic of great importance. When the excitation frequency is close to a natural frequency of the system, within the engine speed working range, the vibration amplitude can lead to high-cycle fatigue failure. Friction damping devices such as UPDs are typically included into turbine design to limit these resonant vibrations (Berruti et al., 2007; Berruti and Maschio, 2012). Each damper is pressed against the blade platforms by the centrifugal force. When relative motion takes place between damper and blade platform, the generated nonlinear friction forces lead to the dissipation of vibrational energy. From an industrial point of view, it is 
fundamental to have effective tools for the design and performance prediction of UPDs. In the last 20 years different authors worked on this topic and proposed several numerical models, they can be found, among the others, in the papers by Panning et al. (2004), Petrov and Ewins (2007), Cigeroglu et al. (2009), and Firrone et al. (2009).

The basic idea is that the calculation of the nonlinear forced response is performed by coupling the UPD to the blades modelled through FE. This coupling is achieved by inserting a contact element between blades and UPD. The contact element is characterized by four parameters, which must be chosen by the user: the friction coefficient, the normal contact stiffness and the tangential contact stiffness in two perpendicular directions.

The problem of solving the nonlinear equation of the forced response in presence of contact forces, depending on the structure displacement, is nowadays completely overcome. The nonlinear forced response can be calculated with high computational efficiency in the frequency domain using the harmonic balance method (HBM) (Petrov, 2004; Charleux et al., 2006; Cigeroglu et al., 2007; Petrov, 2007; Firrone et al., 2009; Siewert et al., 2009; Petrov, 2012).

Being the calculation method well-established, the still open question is how to choose the contact parameters (friction coefficient, normal and tangential contact stiffness) in order to obtain reliable predictions of the forced response. In literature contact parameters are usually tuned by matching the computed forced response function (FRF) with the measured one. This method, however is not a viable procedure at the design stage, where experimental FRFs are not yet available.

This paper presents the numerical results obtained by a numerical code where the damper model, the contact model and the method for solving the equations are aligned with the state of the art. The attention is here focused on the procedure adopted for choosing contact parameters: only those procedures which do not rely on curve fitting between experimental and numerical FRFs are considered. The present paper adopts two such alternative ways to estimate contact parameters: the first is an established analytical model from the literature and the second is based on direct experimental measurements (i.e. hysteresis cycles) on UPDs on a dedicated test rig. The chosen test case is a bladed disk carrying cylindrical UPDs. The comparison between measured FRFs and simulated ones will highlight strengths and limitations of each method used for contact parameter estimation.

\section{Bladed disk with underplatform dampers}

The test case analyzed in this paper is the bladed disk (blisk) shown in Figure 1a. The bladed disk carries between each couple of blades a cylindrical UPD, sketched in Figure 2a and shown in Figure 10a. This blisk and the UPDs were chosen as a test case since they are part of a test rig (Octopus test rig) (Berruti et al., 2011; Firrone and Berruti, 2012) that provides experimental forced response of blisks with UPDs under controlled conditions.
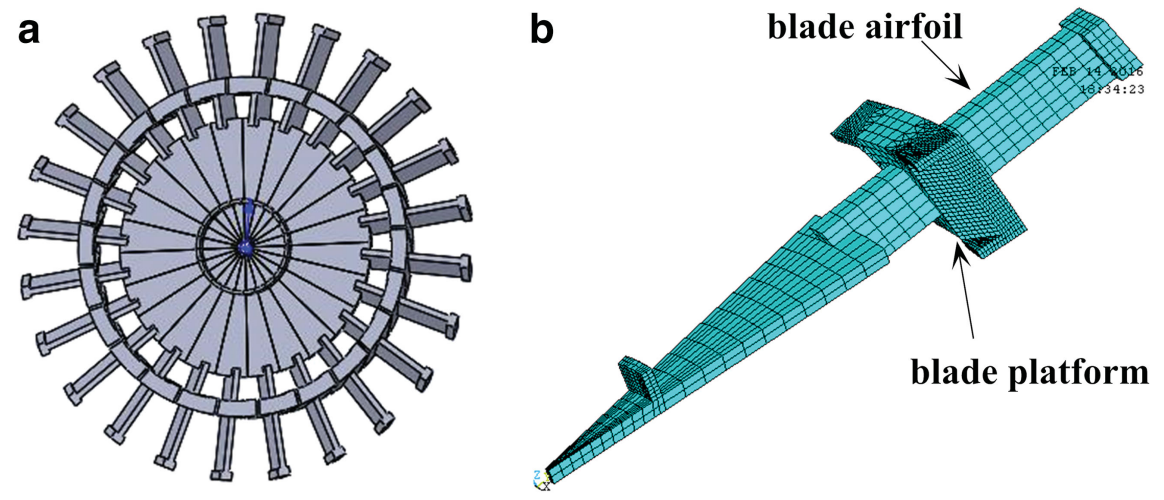

Figure 1. (a) Bladed disk. (b) FE model of a bladed disk sector. 


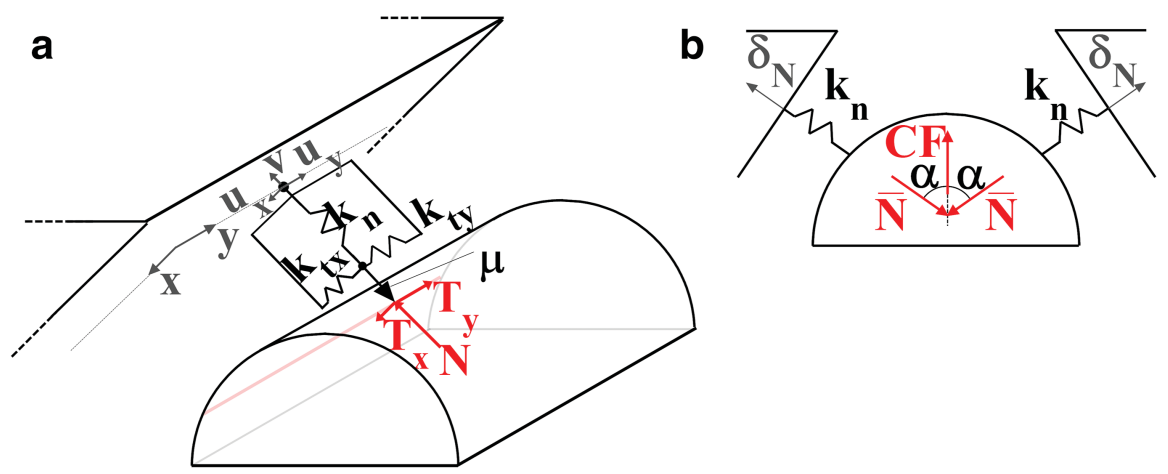

Figure 2. (a) Contact model for a cylindrical UPD. (b) Elastic contact between damper and platform.

\section{Nonlinear dynamic equilibrium equation}

The reduced mass and stiffness matrices of the bladed disk are exported from the FE code in Matlab and they are used for the calculation of the dynamic forced response of the blisk using a dedicated inhouse numerical code.

\section{Dynamic equation of the bladed disk}

The dynamic equations of the bladed disk in the time domain are nonlinear second order differential equations:

$$
\mathbf{M} \ddot{\mathbf{u}}^{*}(\mathrm{t})+\mathbf{C} \dot{\mathbf{u}}^{*}(\mathrm{t})+\mathbf{K u}{ }^{*}=\mathbf{F}_{\mathrm{E}}(\mathrm{t})-\mathbf{F}_{\mathrm{C}}(\mathrm{t})
$$

where $\mathbf{M}, \mathbf{K}$ are the reduced mass and stiffness matrices (Craig and Bampton, 1968), $\mathbf{C}$ is the damping matrix, linear combination of $\mathbf{M}$ and $\mathbf{K}, \mathbf{u}^{*}$ is the vector of the dof (master and slave dofs), $\mathbf{F}_{\mathrm{E}}$ is an engine-order (EO)-type harmonic excitation and $\mathbf{F}_{\mathrm{C}}$ is the vector of nonlinear forces applied by the blade platforms to the cylindrical UPD. The equation can be converted in frequency domain and solved by the HBM. To convert Equation 1 in the frequency domain the displacement and forces are expanded as Fourier series as:

$$
\begin{gathered}
\mathbf{u}^{*}(\mathrm{t})=\mathbf{u}^{*(0)}+\mathrm{R}\left(\sum_{\mathrm{n}=1}^{\mathrm{Nh}} \mathbf{u}^{*(\mathrm{n})} \mathrm{e}^{\mathrm{in} \omega \mathrm{t}}\right) \\
\mathbf{F}_{\mathbf{E}}(\mathrm{t})=\mathbf{F}_{\mathbf{E}}^{(0)}+\mathrm{R}\left(\sum_{\mathrm{n}=1}^{\mathrm{Nh}} \mathbf{F}_{\mathbf{E}}^{(\mathrm{n})} \mathrm{e}^{\mathrm{in} \omega \mathrm{t}}\right) \\
\mathbf{F}_{\mathbf{C}}(\mathrm{t})=\mathbf{F}_{\mathbf{C}}^{(0)}+\mathrm{R}\left(\sum_{\mathrm{n}=1}^{\mathrm{Nh}} \mathbf{F}_{\mathbf{C}}^{(\mathrm{n})} \mathrm{e}^{\mathrm{in} \omega \mathrm{t}}\right)
\end{gathered}
$$

where $\mathrm{Nh}$ is the retained number of harmonics, $\mathrm{n}=0 \ldots \mathrm{Nh}$ and $\omega$ is the frequency of the excitation forces. By substituting Equations 2-3-4 into the general balance Equation 1, the initial nonlinear second order differential equations are turned in the frequency domain into a set of nonlinear algebraic complex equations:

$$
\mathbf{D}(\omega)^{(\mathrm{n})} \mathbf{u}^{*(\mathrm{n})}=\mathbf{F}_{\mathrm{E}}^{(\mathrm{n})}-\mathbf{F}_{\mathrm{C}}^{(\mathrm{n})}
$$

where $\mathbf{D}^{(\mathrm{n})}=-(\mathrm{n} \omega)^{2} \mathbf{M}+\mathrm{in} \omega \mathbf{C}+\mathbf{K}$ is the nth dynamic stiffness matrix of the bladed disk with $\mathrm{n}=$ 0 ...Nh. 


\section{Dynamic equations of the damper}

The damper is considered as a rigid body with inertia properties. The damper equilibrium equation in the time domain is:

$$
\mathbf{M}_{\mathrm{D}} \ddot{\mathbf{h}}(\mathrm{t})=\mathbf{F}_{\mathrm{C}}(\mathrm{t})+\mathbf{F}_{\mathrm{E}-\mathrm{D}}
$$

where $\mathbf{h}$ is the vector of displacements of the damper center of mass, $\mathbf{F}_{C}(t)$ is the vector of components of the resultant of the contact forces at the mass center, $\mathbf{F}_{\mathrm{E}-\mathrm{D}}=\{0, \mathrm{CF}, 0,0,0,0\}$ is the vector of components of the external forces, the only external force on the damper is $\mathrm{CF}$, the centrifugal force. $\mathbf{M}_{\mathrm{D}}$ is the damper mass matrix. By following the same procedure carried out for the bladed disk case, Equation 6 can be solved in the frequency domain as:

$$
\mathbf{D}_{\mathrm{D}}(\omega)^{(\mathrm{n})} \mathbf{h}^{(\mathrm{n})}=\mathbf{F}_{\mathrm{E}-\mathrm{D}}^{(\mathrm{n})}+\mathbf{F}_{\mathrm{C}}^{(\mathrm{n})}
$$

where $\mathrm{Nh}$ is the retained number of harmonics, $n=0 \ldots N$ and $\mathbf{D}_{D}{ }^{(n)}=-(n \omega)^{2} \mathbf{M}_{D}$ is the $n^{\text {th }}$ dynamic stiffness matrix of the damper. In order to solve Equations 5 and 7, a contact model is introduced to compute the contact forces $\mathbf{F}_{\mathrm{C}}$ that are unknown. The contact forces depend on the relative displacement between damper and blade platforms, this dependence makes the Equations 5 and 7 nonlinear. As a consequence, an iterative solution method like the Newton-Raphson solver has to be used.

\section{Contact model}

The contact model, a typical macroslip element, takes into account a two-dimensional tangential relative displacement and a variable normal load. The contact model applied to a contact point on the damper is sketched in Figure 2a.

The plane $(x, y)$ is tangent to the UPD and represents the surface of the blade platform. Two springs define the tangential contact stiffnesses $\mathrm{k}_{\mathrm{tx}}$ and $\mathrm{k}_{\mathrm{ty}}$ along the two orthogonal directions $\mathrm{x}$ and $\mathrm{y}$. The normal contact stiffness is represented by the spring with stiffness $k_{n}$. The two parameters $\mathrm{u}(\mathrm{t})=\left\{\mathrm{u}_{\mathrm{x}}(\mathrm{t}) ; \mathrm{u}_{\mathrm{y}}(\mathrm{t})\right\}$ and $\mathrm{v}(\mathrm{t})$ are the periodic relative displacements of the contact nodes in the tangential and normal directions respectively. $\mathrm{T}_{\mathrm{x}}, \mathrm{T}_{\mathrm{y}}$ and $\mathrm{N}$ are the contact friction forces generated as a result of the relative motion between platform and damper.

\section{Determination of contact parameters}

A key point for a correct calculation of the forced response in presence of UPDs is the choice of the correct contact parameters (contact stiffness and friction coefficient) to be given as input to the contact model.

There are analytical methods in literature (Brändlein et al., 1999; Harris and Kotzalas, 2006; Allara et al., 2008; Allara, 2009) to calculate the contact stiffness values in normal and tangential direction starting from the geometry and the material of the UPD. The same contact stiffness values can also be determined from measurement of forces and displacement but only on dedicated test rigs.

Some focus on single flat-on-flat contacts under constant normal loads and high temperatures (Lavella et al., 2011; Botto et al., 2012; Lavella, 2016), and are therefore not useful in this specific case. On the other hand, the Damper Test Rig (Gola and Liu, 2014) aims at estimating contact parameters of UPDs (cylinder-on-flat interfaces as well) under more realistic working conditions in terms of variable normal load and complex interface kinematics.

The friction coefficient cannot be calculated with the analytical technique presented here, it is therefore measured. These different approaches for the choice of the contact parameter values are here presented. 


\section{Analytical calculation of the contact stiffness values \\ Normal contact stiffness}

The normal load $\mathrm{N}$ at the contact line causes an elastic deformation $\delta_{\mathrm{N}}$ due to the elastic contact stiffness $\mathrm{k}_{\mathrm{n}}$. The calculation of the normal contact stiffness can be performed applying formulas found in literature. The well-known Harris (Harris and Kotzalas, 2006) empirical formula allows the calculation of the normal elastic penetration $\delta_{\mathrm{N}}$ occurring when a cylinder is pressed against a flat surface (steel on steel):

$$
\delta_{\mathrm{N}-\text { Harris }}=3.84 \cdot 10^{-5} \frac{\mathrm{N}^{0.9}}{\mathrm{~L}^{0.8}}
$$

where $\mathrm{N}$ is the value of the normal contact force and $\mathrm{L}$ is the length of the cylinder minus possible recesses. The same contact condition was more recently studied by Brändlein et al. (1999) who established that the normal elastic penetration for the single contact is obtained as:

$$
\delta_{\mathrm{N}-\mathrm{B}}=4.05 \cdot 10^{-5} \frac{\mathrm{N}^{0.925}}{\mathrm{~L}^{0.85}}
$$

Allara et al. (2008) and Allara (2009) use the pressure distribution to compute the normal relative displacements of the contact surfaces by means of the Cerruti potential theory (Hills et al., 1993):

$$
\delta_{\mathrm{N}-\text { Allara }}=\frac{2\left(1-v^{2}\right)}{\pi \mathrm{E}} \frac{\mathrm{N}}{\mathrm{L}}\left[1+\ln \left|\frac{2 \mathrm{~L}}{\mathrm{~b}}\right|^{2}\right]
$$

where $\mathrm{b}$ is the half-width of the contact area, $\mathrm{E}$ is the Young's modulus and $v$ is the Poisson's ratio. The value of the normal contact stiffness is obtained by taking the derivative of the normal penetration $\delta_{\mathrm{N}}$ with respect to $\mathrm{N}$ :

$$
\frac{1}{\mathrm{k}_{\mathrm{n}}(\mathrm{N})}=\frac{\mathrm{d} \delta_{\mathrm{N}}}{\mathrm{dN}}
$$

Hence,

$$
\begin{gathered}
\mathrm{k}_{\mathrm{n}-\text { Harris }}(\mathrm{N})=\frac{10}{9} \frac{\mathrm{L}^{0.8}}{3.84 \cdot 10^{-5}} \mathrm{~N}^{0.1} \\
\mathrm{k}_{\mathrm{n}-\mathrm{B}}(\mathrm{N})=\frac{1}{0.925} \frac{\mathrm{L}^{0.85}}{4.05 \cdot 10^{-5}} \mathrm{~N}^{0.075} \\
\mathrm{k}_{\mathrm{n}-\text { Allara }}(\mathrm{N})=\frac{\pi \mathrm{EL}}{2\left(1-v^{2}\right) \ln \left(\frac{2 \mathrm{~L}}{\mathrm{~b}}\right)^{2}}
\end{gathered}
$$

In the last equation, the dependence on the normal load $\mathrm{N}$ is hidden in $\mathrm{b}$, half-width of the contact area, function of $\mathrm{N}$.

Equation 12-14, are here applied to the cylindrical UPD of Figure $2 \mathrm{a}$ where the length is $\mathrm{L}=27.6 \mathrm{~mm}$ and the radius of the cylinder is $\mathrm{R}=5 \mathrm{~mm}$. The length of contact corresponds to the nominal length of the UPD, as shown by the wear marks (see Results section). The half-width of the contact area $\mathrm{b}$ can be calculated by the standard Hertz formulas (Harris and Kotzalas, 2006; Allara et al., 2008). Figure 3 shows the plot of the normal contact stiffness calculated according to the Equation 12-14 versus the normal load $\mathrm{N}$.

Figure 3 shows that the three analytical models lead to three different plots. It will be observed later however that this discrepancy does not significantly influence the calculated FRFs of blades with damper: it leads to a difference lower than $1.5 \%$ in the frequency value of the blades with damper when the different $\mathrm{k}_{\mathrm{n}}$ are used. For all the subsequent calculations it was then decided to use one of them, Brändlein's formulation of $\mathrm{k}_{\mathrm{n}}$ (Equation 13) was chosen. 


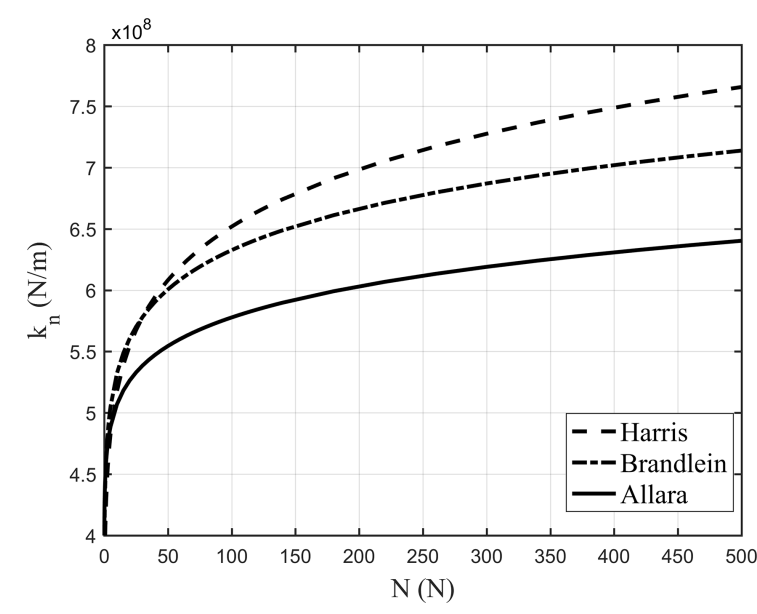

Figure 3. Normal contact stiffness as a function of the normal load for different analytical models.

\section{Tangential contact stiffness}

The cylindrical UPD, in contact with the blade platforms, undergoes contact forces and displacements in the tangential direction.

Allara et al. (2008) and Allara (2009) found the analytical expression for the monotonic loading curves and hysteresis cycles (i.e. contact forces vs. relative displacement) of a filleted flat punch pressed against an infinite half-plane. The flat punch can be customized with material properties and contact area width, length and fillet radius. As shown in Figure 4 this parameter setting can be used to reproduce the case of a cylinder pressed against a plane moving alternatively along the $\mathrm{x}$ direction (Figure 4a) and the y direction (Figure 4b) to determine respectively $\mathrm{k}_{\mathrm{tx}}$ and $\mathrm{k}_{\mathrm{ty}}$.

\section{Experimental estimation of the contact stiffness values}

Contact stiffness values $\mathrm{k}_{\mathrm{n}}, \mathrm{k}_{\mathrm{tx}}$ are here derived from measurements using a test rig (Gola and Liu, 2014; Gastaldi and Gola, 2016a,b) purposely developed for the direct experimental investigation of the contact stiffness in UPDs. The experimental evidence used here comes from an experimental campaign on a three-point laboratory damper, shown in Figure 5. This three-point damper shares with the cylindrical damper of the Octopus test rig the same material, curvature radius and contact pressure. Contact stiffness values can therefore be safely deduced using the experimental evidence on the threepoint damper.

The test rig shown in Figure 6a, developed over the years by the AERMEC laboratory (see Gola and Liu, 2014 for a detailed description), is designed aiming at:

1. imposing user-defined in-plane displacements simulating the so-called In-Phase (IP, vertical) and Out-of-Phase (OOP, horizontal) relative motion between the blades platforms by means of two piezo-actuators connected to the left dummy platform;

2. measuring the forces transmitted between the two platforms through the damper by means of two load cells connected to the right dummy platform.

Please notice that all measured quantities are reported both in Figure 5 and in Figure 6a. Relative platforms displacements, measured using a laser head, are plotted against the corresponding component of the contact force (" $h_{L P}$ vs. $H_{R}$ " in case of OOP motion and " $w_{L P} v s$. $W_{R}$ " in case of IP motion) in the platform-to-platform damper hysteresis cycle. The evolution of a typical OOP hysteresis cycle is shown in Figure $6 \mathrm{~b}$. The slope $\mathrm{k}_{\mathrm{H}}$ highlighted in Figure $6 \mathrm{~b}$ keeps constant throughout the evolution and corresponds to a specific contact state: all contact points (R, L1 and L2) are

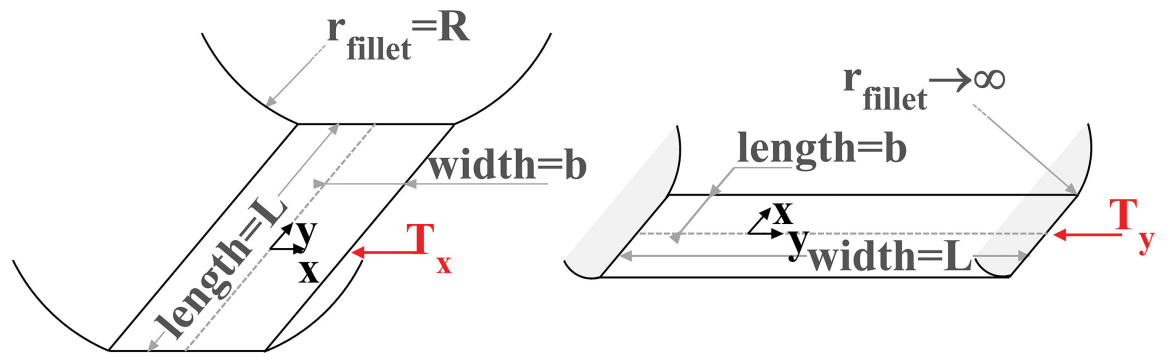

Figure 4. Allara flat punch model. 


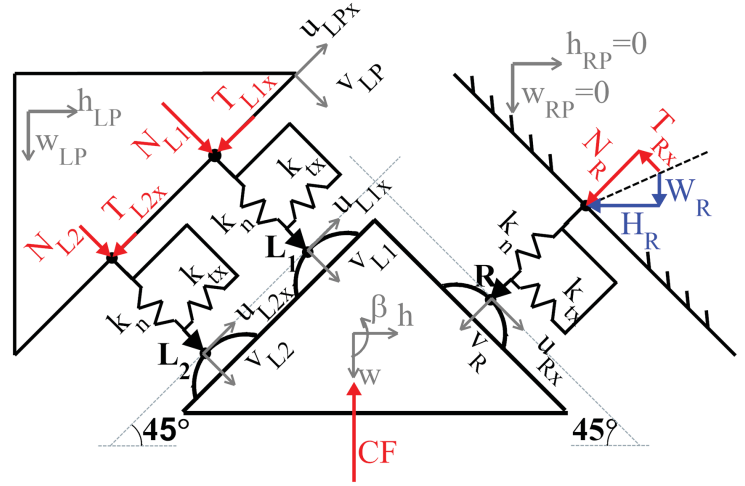

Figure 5. Damper test rig set up and relevant quantities.

different values of $\mathrm{k}_{\mathrm{ty}}$ had on the position and amplitude of the peak of the full-stick FRF. Table 1 lists the results of this investigation (each value was obtained using $\mathrm{k}_{\mathrm{tx}}$ and $\mathrm{k}_{\mathrm{n}}$ estimated starting from measurements as described above). Sensitivity is even lower when frequency peaks of blades/UPDs systems undergoing slip are considered.

It can be observed that the sensitivity of frequency and amplitude of the stuck peak to variations of $\mathrm{k}_{\mathrm{ty}}$ is low (a variation of two orders of magnitude on $\mathrm{k}_{\mathrm{ty}}$ produces an increment lower than $4.5 \%$ and $3.5 \%$ respectively), especially if compared to variations of $\mathrm{k}_{\mathrm{tx}}$ (see Table 2 and Figure 12 vs. Figure 14). Since the effect of the dispersion of experimental data (considering both repeatability and bladeto-blade difference) is comparable in terms of peak frequency and much larger in terms of amplitude, the lack of knowledge on $\mathrm{k}_{\mathrm{ty}}$ is considered not critical for the investigation of the first bending mode. Its influence may be stronger if a torsional mode was considered. In that case an additional experimental investigation to determine the true value of $\mathrm{k}_{\mathrm{ty}}$ will be needed. In the present case it was decided to set $\mathrm{k}_{\mathrm{ty}}=\mathrm{k}_{\mathrm{tx}}$.

\section{Determination of the friction coefficient}

The value of the friction coefficient was measured through ad hoc experimental measurements as described by Gastaldi and Gola (2016a). A value of 0.6 was measured at room temperature over a

a

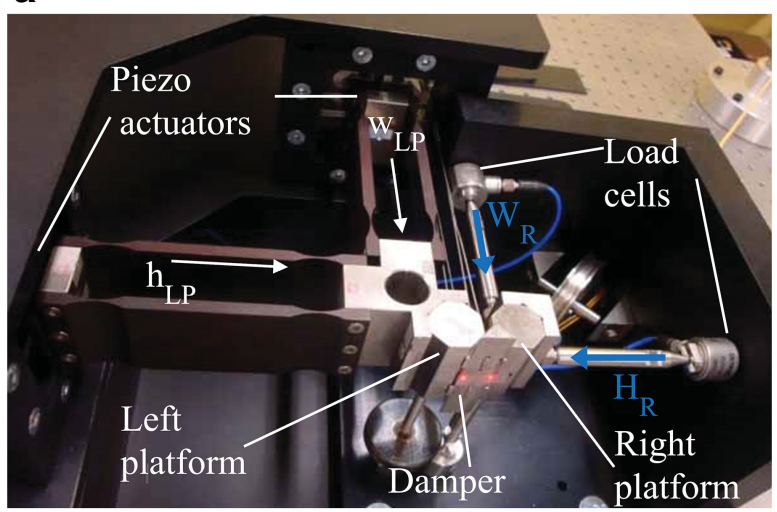

b

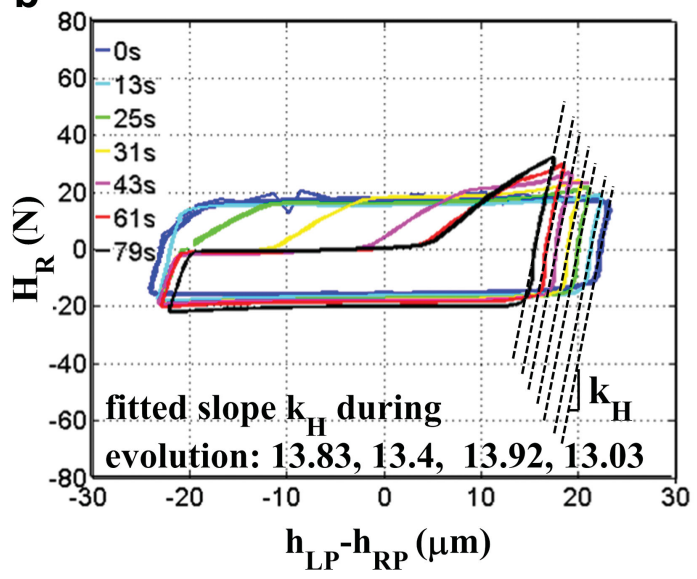

Figure 6. (a) Damper test rig photo and measured quantities, displacements in white, forces in blue. (b) Example of hysteresis cycle time evolution. 
Table 1. Frequency values in stuck condition using contact stiffness values estimated experimentally.

\begin{tabular}{|l|c|c|}
\hline $\mathrm{k}_{\mathrm{ty}}$ Tentative value & Stuck peak frequency $(\mathrm{Hz})$ & Stuck peak amplitude (m/s/N) \\
\hline $\mathrm{k}_{\mathrm{ty}}=0.1 \cdot \mathrm{k}_{\mathrm{tx}}$ & 143.8 & 0.548 \\
\hline $\mathrm{k}_{\mathrm{ty}}=\mathrm{k}_{\mathrm{tx}}$ & 144.9 & 0.531 \\
\hline $\mathrm{k}_{\mathrm{ty}}=10 \cdot \mathrm{k}_{\mathrm{tx}}$ & 150.3 & 0.528 \\
\hline
\end{tabular}

Table 2. Total contact stiffness.

\begin{tabular}{|l|c|c|c|}
\hline Method & $\mathrm{k}_{\mathrm{n}}$ & $\mathrm{k}_{\mathrm{tx}}$ & $\mathrm{k}_{\mathrm{ty}}$ \\
\hline Analytical & $5.50 \cdot 10^{8}$ & $5.71 \cdot 10^{8}$ & $6.07 \cdot 10^{8}$ \\
\hline From measurements & $5.53 \cdot 10^{8}$ & $1.70 \cdot 10^{7}$ & $1.70 \cdot 10^{7}$ \\
\hline
\end{tabular}

range of $5 \cdot 10^{6}$ cycles. Furthermore, the measurements in (Lavella et al., 2013) match with the stabilized values of friction coefficients for cylindrical contacts measured on the dedicated damper rig (Gastaldi and Gola, 2016a).

\section{Experimental Results}

Experimental results of the forced response measurements are available from the Octopus test rig described by Berruti et al. (2011) and Firrone et al. (2012). A selection of experimental FRFs are here compared with the calculation results in order to check whether each choice of contact parameters is correct.

\section{Description of the test rig}

Figure 7 a shows the Octopus test rig. The bladed disk (1), is fixed to a support (2). The arm structures (3) hold one pulley each, they are mounted on the external ring (4) equally spaced around the circumferential disk direction. The simulation of the centrifugal force on each UPD is obtained by means of two wires (5), which pass over the arms and are connected through the pulley to a dead weight. The excitation system is a non-contact travelling wave generated by electromagnets below each blade as shown in Figure $7 \mathrm{~b}$.

The electromagnets can be activated with a given phase shift in time in order to generate the required EO type excitation. The dynamic response of the disk is carried out by means of a laser scanning vibrometer. An example of measurement is shown in Figure 8 which shows the FRFs of the 24 blades excited by $\mathrm{EO}=2$, excitation force on each blade $\mathrm{F}_{\text {ext }}=0.3 \mathrm{~N}$ (where $\mathrm{F}_{\text {ext }}=\left|\mathrm{F}_{\mathrm{E}}\right|$ of Equation 5) and centrifugal force on each UPDs CF $=147 \mathrm{~N}$, for two sets of different measurements (Measure I and Measure II). The second set of measurements (Measure II) is repeated after mounting and dismounting of the damper.

\section{Post-processing of measured mistuned responses}

It can be seen in Figure 8 that, due to the presence of small mistuning, defined as small variations between each disk sector as explained by Castanier and Pierre (2006), there is a difference between the FRFs of each blade. The bold red and blue lines are the envelope of the maxima of the FRFs at every frequency for the two sets of measurements. The envelope of the maxima represents the worst case working condition that is 

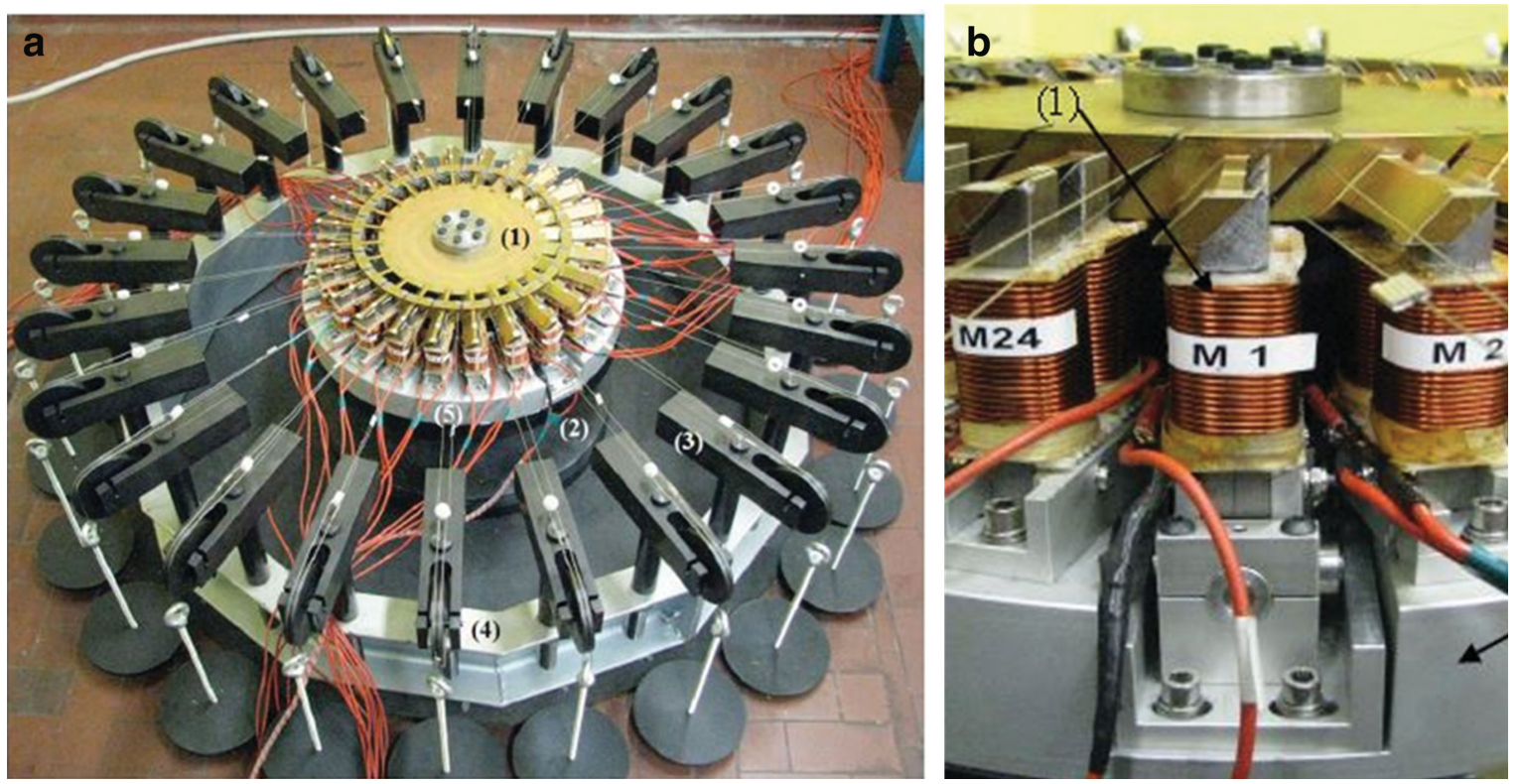

Figure 7. (a) The test rig Octopus. (b) Detail of the excitation system.

the maximum vibration amplitude for each frequency. The two curves representing the envelope of the maxima are not overlapped but they show that the maximum amplitude value and the resonance frequency value is in the same range for the two set of measurements. Similar FRFs are obtained for different excitation amplitude values. The two curves of the envelope of the maxima (an example is Figure 8) will be used as reference for comparison with numerical results.

\section{Results and discussion}

The numerical procedure described in the previous sections has been applied for the calculation of the forced response of the first bending mode (excitation type $\mathrm{EO}=2$ ) of the bladed disk. A numerical model becomes predictive thanks to a combination of factors. A representative FE model, the faithful representation of the boundary conditions and a numerical solver adequate to the application are considered as prerequisites. The soundness of the techniques mentioned above is demonstrated by the good matching between the numerical and experimental response of the disk without UPDs (i.e. linear case in Figure 9).

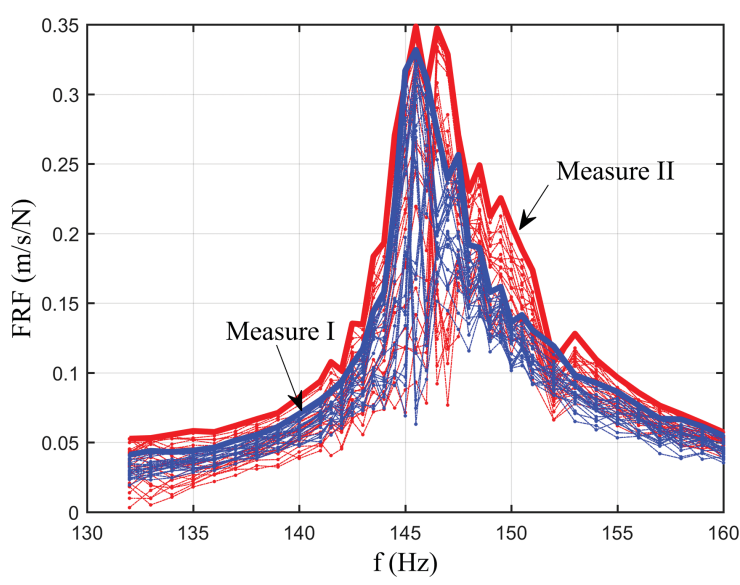

Figure 8. FRFs of the 24 blades, $\mathrm{EO}=2$, Fext $=0.3$ $\mathrm{N} \mathrm{CF}=147 \mathrm{~N}$, for two sets of measurements.
Other factors such as a trustworthy representation of contact conditions, achieved only through a deep knowledge of the contact parameters, requires instead a novel approach, which is the focus of this paper.

The calculation with the UPDs requires the selection of contact nodes to which macroslip elements (contact forces) are applied. From the observation of the wear marks on the damper (as shown in Figure 10a) it was deduced that the contact was along all the contact line cylinderplatform. The contact nodes were then selected along a line on the platform as shown in Figure 10b. A contact model like that shown in Figure 2a was then applied to each contact node. The crucial point is the choice of the correct contact parame- 


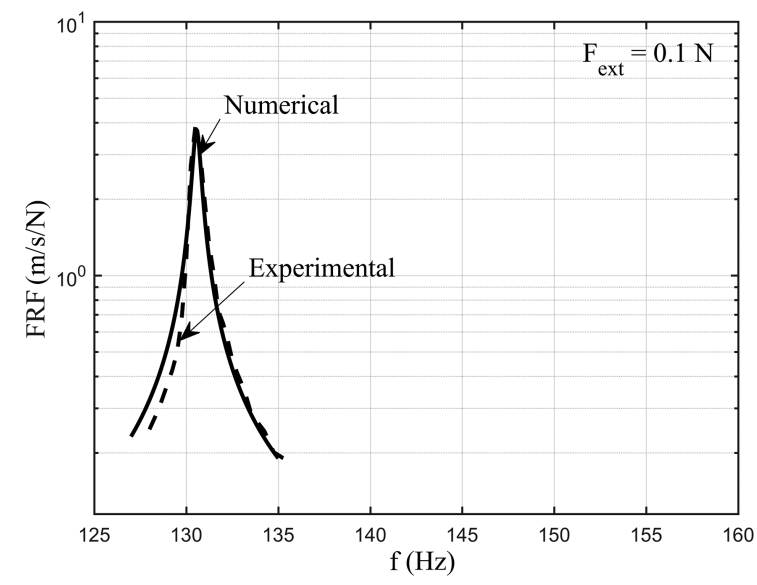

Figure 9. Comparison between experimental and numerical FRFs. $E O=2$. Bladed disk without UPDs.

fied by the results of the simulation: the first harmonic component of $\mathrm{N}$ is one order of magnitude smaller than the static component $\overline{\mathrm{N}} \cdot \overline{\mathrm{N}}$ can be estimated under the assumption that the static components of the tangential contact force $\bar{T}_{x}$ and $\bar{T}_{y}$ are close to zero (a reasonable assumption given the inversion of motion the damper undergoes during harmonic oscillation). As shown in Figure $2 \mathrm{~b}, \overline{\mathrm{N}}$ is expressed as a function of the centrifugal force CF applied to the damper center of mass and of the platform angle $\alpha$ :

$$
\overline{\mathrm{N}}=\frac{\mathrm{CF}}{2 \cos (\alpha)}
$$

On the same Table 2 in the second row, the contact stiffness values, coming from the data treatment of the measurements on the rig of Figure 6 , are listed. The value of $\mathrm{k}_{\mathrm{ty}}$ was assumed equal to $\mathrm{k}_{\mathrm{tx}}$ since it was not measured on the test rig. It can be observed that the calculated normal stiffness $\left(k_{n}\right)$ value matches with the measured value. On the contrary, the calculated tangential stiffness $\left(\mathrm{k}_{\mathrm{tx}}\right)$ is an order of magnitude lower the measured one. The FRF calculation results using as input the calculated stiffness values (first row of Table 2) are shown in Figure 11. The other input parameters are: centrifugal force on the damper is $\mathrm{CF}=147 \mathrm{~N}, \mathrm{EO}=2$, first bending mode, excitation force on each blade $\mathrm{F}_{\text {ext }}=0.2 \mathrm{~N}$.

Figure 12 shows the results for the same case but with $\mathrm{F}_{\mathrm{ext}}=0.4 \mathrm{~N}$. It can be observed how the calculated value of contact stiffness leads to FRFs where the resonance frequency is largely overestimated. The calculated FRF does not match with the experimental FRFs neither in terms of frequency nor in terms of amplitude. On the other hand, if the contact stiffness values coming from the measurement (second row of Table 2) are used as input in the calculation, the FRFs of Figure 13 and Figure 14 for the same two cases are obtained.
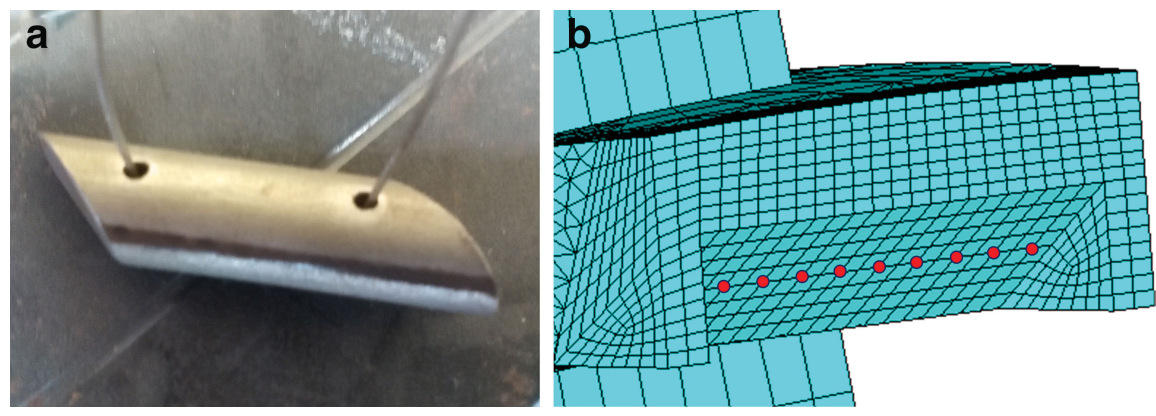

Figure 10. (a) Wear marks on the damper. (b) Selection of contact nodes. 


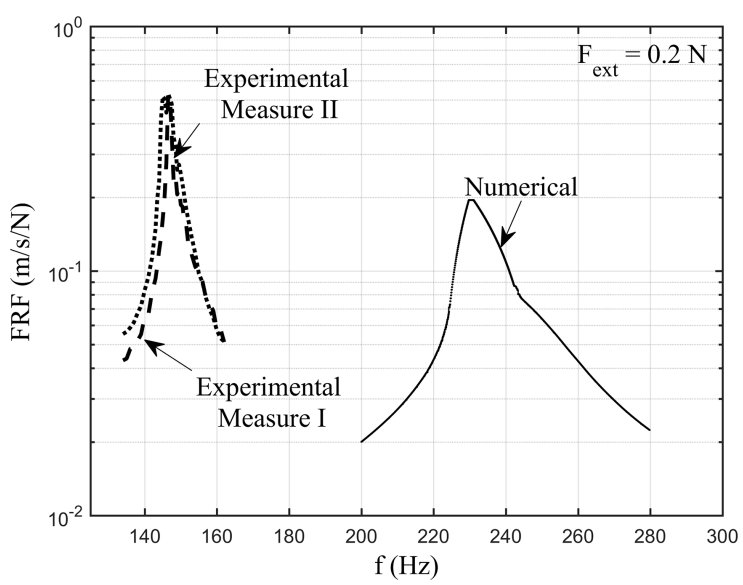

Figure 11. Comparison between experimental and numerical FRFs. Fext $=0.2 \mathrm{~N}$. Contact stiffness values estimated analytically.

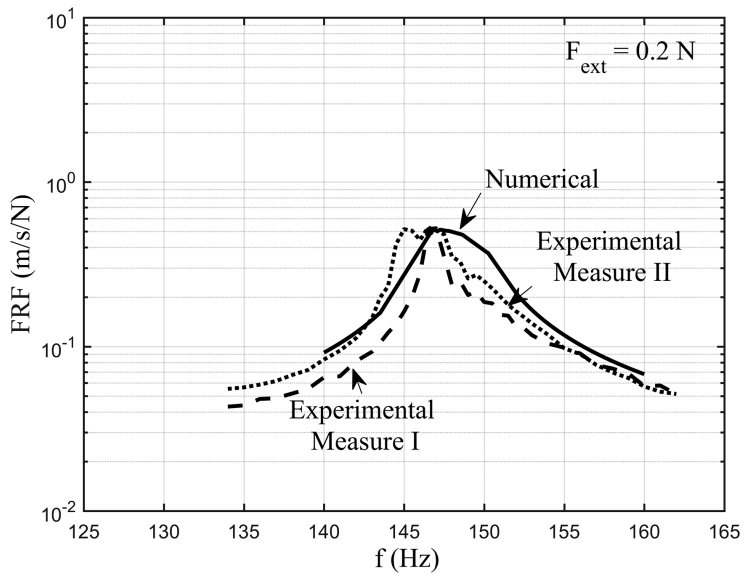

Figure 13. Comparison between experimental and numerical FRFs. Fext $=0.2 \mathrm{~N}$. Contact stiffness values estimated through direct experiments on dampers.

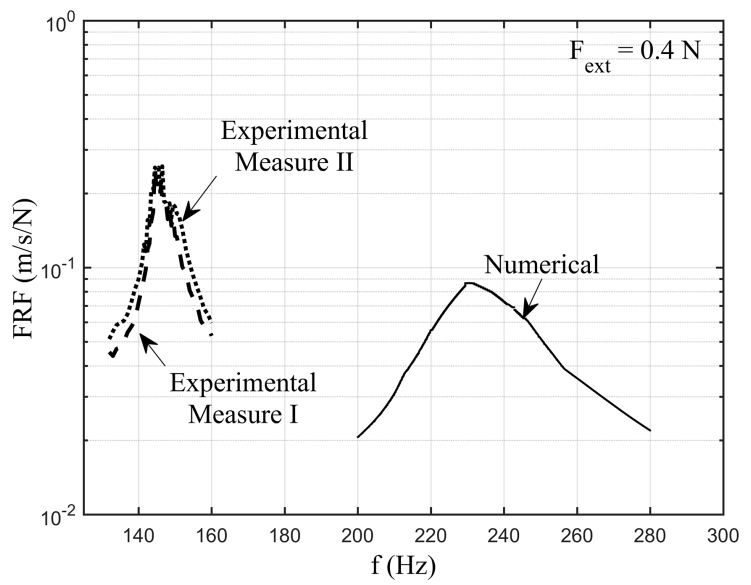

Figure 12. Comparison between experimental and numerical FRFs. Fext $=0.4 \mathrm{~N}$. Contact stiffness values estimated analytically.

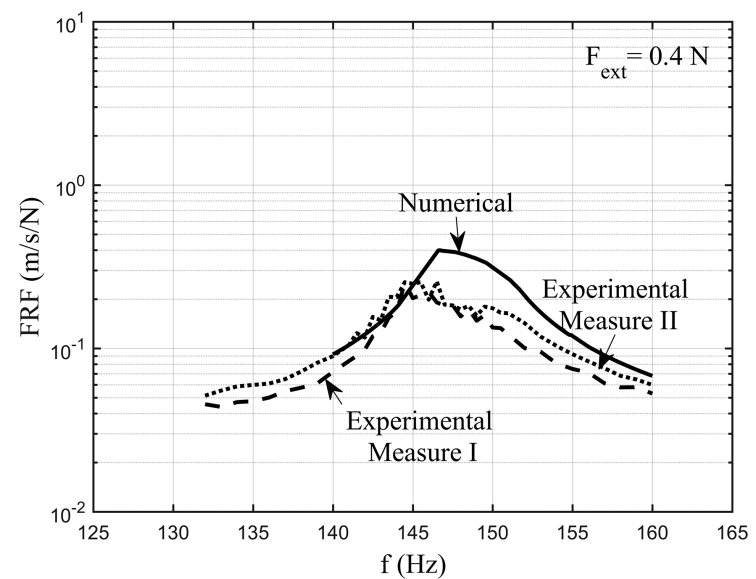

Figure 14. Comparison between experimental and numerical FRFs. Fext $=0.4 \mathrm{~N}$. Contact stiffness values estimated through direct experiments on dampers.

It can be observed how in this case the calculated FRFs match quite satisfactorily their experimental counterparts in terms of amplitude, shape of the peak and peak frequency. The main difference is in the value of $\mathrm{k}_{\mathrm{tx}}$. The measured value of $\mathrm{k}_{\mathrm{tx}}$ is one order of magnitude lower than that calculated analytically and it proves to be the correct value. The analytical method, which simulates a flat punch with a fillet radius pressed against a plane, is not fit to simulate a cylindrical contact since it gives a too high tangential contact stiffness value. This may be due to the fact that the method does not allow for micro-rotation or to the fact that it does not allow for variation of normal load. Both phenomena are instead encountered by the UPD during working condition.

\section{Conclusions}

The paper investigates two different ways to choose the contact stiffness values for the contact model of UPDs. Contact stiffness values can be derived from analytical modles available in literature (method 1) or they can be derived from measurements on a dedicated test rig with a laboratory damper, but with similar working conditions of the damper under study (method 2). 
The investigation is completed by the experimental/numerical comparison of FRFs of a blisk with cylindrical UPDs. As mentioned above, numerical FRFs have been obtained using different contact stiffness values obtained with two different methodologies. It should be noted that in this paper experimental/numerical comparison of FRFs is not used as a mean to tune the model, but as a separate and independent tool to evaluate different methods for contact parameter estimation.

Regarding the normal contact stiffness $\mathrm{k}_{\mathrm{n}}$ the analytical calculation values (Brändlein, Harris, Allara) and the value derived from measurements on the laboratory damper agree. The small discrepancies between the obtained values have negligible effects over the forced response (i.e. well below the experimental FRF dispersion).

Regarding the tangential contact stiffness $\mathrm{k}_{\mathrm{tx}}$ (in the plane perpendicular to the damper axis), Allara's analytical method (method 1 ) gives a value of the tangential contact stiffness $\mathrm{k}_{\mathrm{tx}}$ one order of magnitude higher than the value obtained by the derivation from measurements on the laboratory damper. In the calculation of the FRF this $\mathrm{k}_{\mathrm{tx}}$ value leads to a discrepancy $>50 \%$ between the measured and simulated peak frequency. Allara's analytical method, which simulates a flat punch with a fillet radius pressed against a plane, is clearly not fit to simulate a cylindrical contact. On the contrary, the method, based on direct measurements of $\mathrm{k}_{\mathrm{tx}}$ on a dedicated test rig with a laboratory damper (method 2) produces a value of $\mathrm{k}_{\mathrm{tx}}$ one order of magnitude lower than that calculated analytically. This value of measured $\mathrm{k}_{\mathrm{tx}}$, given to the calculation code, was successful in producing matching numerical FRFs.

In these authors opinion, the method was successful because the working conditions of the two dampers, the one tested on the dedicated test rig and the one under study, are comparable (same contact geometry, pressure, material and kinematics) and the data processing technique takes into account the difference of position and number of contact points.

These authors are therefore confident on the determination of the contact stiffness, rather than through analytical model, though careful and direct measurements on dedicated test rigs.

The results obtained here proved that measurements of contact stiffness on laboratory dampers under controlled conditions can be exported and used on other dampers.

\section{Appendix A}

It is here summarized how the values of the contact stiffness $\mathrm{k}_{\mathrm{n}}$ and $\mathrm{k}_{\mathrm{tx}}$ are derived from the experimental measurements on the test rig of Figure 6.

Consider the damper equilibrium in stick condition:

$$
\mathbf{M}_{\mathbf{D}} \ddot{\mathbf{h}}=\mathbf{F}_{\mathbf{E}-\mathbf{D}}+\mathbf{T F}_{\mathrm{C}}
$$

where $\mathbf{M}_{\mathbf{D}}$ is the damper mass matrix, $\mathbf{h}=\{\mathrm{h}, \mathrm{w}, \beta\}$ is the vector of damper displacements in global coordinates, $\mathbf{F}_{\mathbf{E}-\mathbf{D}}=\{0, \mathrm{CF}, 0\}$ is the vector of external forces, $\mathbf{F}_{\mathbf{C}}=\left\{\mathrm{T}_{\mathrm{R}}, \mathrm{N}_{\mathrm{R}}, \mathrm{T}_{\mathrm{L} 1}, \mathrm{~N}_{\mathrm{L} 1}, \mathrm{~T}_{\mathrm{L} 2}, \mathrm{~N}_{\mathrm{L} 2}\right\}$ is the vector of contact forces and $\mathbf{T}$ is a $6 \times 3$ transformation matrix. It should be noted that the notation in Equation 16 is compatible with that in Equations 6 and 7. In Equation 16 however only 3 out of 6 rigid body motion parameters are considered to reproduce the in-plane damper test rig set up (see Figure $7 \mathrm{a})$.

The vector $\mathbf{F} \mathbf{c}$ in case of full stick can be expressed as:

$$
\mathbf{F}_{\mathrm{C}}=\left[\begin{array}{cccccc}
\mathrm{k}_{\mathrm{tx}} & 0 & \ldots & \ldots \\
0 & \mathrm{k}_{\mathrm{n}} & & & \\
\vdots & \mathrm{k}_{\mathrm{tx}} & 0 & \vdots \\
& 0 & \mathrm{k}_{\mathrm{n}} & \vdots \\
\ldots & \ldots & \mathrm{k}_{\mathrm{tx}} & 0 \\
& & & & 0 & \mathrm{k}_{\mathrm{n}}
\end{array}\right]\left(\left\{\begin{array}{c}
\mathrm{u}_{\mathrm{Rx}} \\
\mathrm{v}_{\mathrm{R}} \\
\mathrm{u}_{\mathrm{L} 1 \mathrm{x}} \\
\mathrm{v}_{\mathrm{L} 1} \\
\mathrm{u}_{\mathrm{L} 2 \mathrm{x}} \\
\mathrm{v}_{\mathrm{L} 2}
\end{array}\right\}-\left\{\begin{array}{c}
\mathrm{u}_{\mathrm{RPx}} \\
\mathrm{v}_{\mathrm{RP}} \\
\mathrm{u}_{\mathrm{LPx}} \\
\mathrm{v}_{\mathrm{LP}} \\
\mathrm{u}_{\mathrm{LPx}} \\
\mathrm{v}_{\mathrm{LP}}
\end{array}\right\}\right)=\mathbf{K}\left(\mathbf{u}-\mathbf{u}_{\mathbf{P}}\right)
$$


Using transformation matrices $\mathbf{T}^{\mathbf{3}}$ and $\mathbf{T}_{\mathbf{P}}$ ' to switch between local and global coordinate system of damper and platforms respectively:

$$
\mathbf{F}_{\mathbf{C}}=\mathbf{K}\left(\mathbf{T}^{\prime} \mathbf{h}-\mathbf{T}_{\mathbf{P}}{ }^{\prime} \mathbf{h}_{\mathbf{P}}\right)
$$

The equilibrium Equation 16 can be transformed in its incremental form:

$$
\mathbf{M} \Delta \ddot{\mathbf{h}}=\mathbf{T} \Delta \mathbf{F}_{\mathbf{C}}
$$

By neglecting the damper variation of inertial forces $\left(\Delta \mathbf{F}_{C}=0\right)$ it is possible to express the damper displacements as a function of the platform displacements. With reference to Equation 18, it holds:

$$
\Delta \mathbf{h}=\mathbf{T T}_{\mathbf{P}}{ }^{\prime} \Delta \mathbf{h}_{\mathbf{P}}
$$

Let us now express the variation of contact forces as a function of the variation of platform displacements by substituting Equation 20 in Equation 18:

$$
\Delta \mathbf{F}_{\mathbf{c}}=\mathbf{K}\left(\mathbf{T}^{\prime} \mathbf{T} \mathbf{T}_{\mathbf{P}}{ }^{\prime}-\mathbf{T}_{\mathbf{P}}{ }^{\prime}\right) \Delta \mathbf{h}_{\mathbf{P}}
$$

The last step involves isolating the horizontal and vertical components of the right contact force $\left(\mathrm{H}_{\mathrm{R}}\right.$ and $\mathrm{V}_{\mathrm{R}}$ ) using a transformation matrix termed here $\mathrm{T}_{\mathrm{HV}}$.

$$
\begin{gathered}
\left\{\begin{array}{c}
\Delta \mathrm{H}_{\mathrm{R}} \\
\Delta \mathrm{V}_{\mathrm{R}}
\end{array}\right\}=\mathbf{T}_{\mathbf{H V}} \Delta \mathbf{F}_{\mathbf{c}}=\mathbf{T}_{\mathbf{H V}} \mathbf{K}\left(\mathbf{T}^{\prime} \mathbf{T} \mathbf{T}_{\mathbf{P}}{ }^{\prime}-\mathbf{T}_{\mathbf{P}}{ }^{\prime}\right) \Delta \mathbf{h}_{\mathbf{P}}=\hat{\mathbf{K}} \Delta \mathbf{h}_{\mathbf{P}} \\
\quad=\left[\begin{array}{cccc}
-\mathrm{k}_{\mathrm{H}} & -\mathrm{k}_{\mathrm{HV}} & \mathrm{k}_{\mathrm{H}} & \mathrm{k}_{\mathrm{HV}} \\
\mathrm{k}_{\mathrm{VH}} & -\mathrm{k}_{\mathrm{V}} & \mathrm{k}_{\mathrm{VH}} & \mathrm{k}_{\mathrm{V}}
\end{array}\right]\left\{\begin{array}{c}
\Delta \mathrm{h}_{\mathrm{RP}} \\
\Delta \mathrm{w}_{\mathrm{RP}} \\
\Delta \mathrm{h}_{\mathrm{LP}} \\
\Delta \mathrm{w}_{\mathrm{LP}}
\end{array}\right\}
\end{gathered}
$$

The analytical expression of entries $(1,1)$ and $(1,3)$ of matrix $\hat{\mathbf{K}}$ are set equal to the fitted slope $\mathrm{k}_{\mathrm{H}}$ in Figure $6 \mathrm{~b}$, while entries $(2,2)$ and $(2,4)$ are set equal to $\mathrm{k}_{\mathrm{V}}$. The solution of the system

$$
\left\{\begin{array}{l}
\hat{\mathbf{K}}(1,3)=\mathrm{k}_{\mathrm{H}} \\
\hat{\mathbf{K}}(2,4)=\mathrm{k}_{\mathrm{V}}
\end{array}\right.
$$

is unique and allows determining $\mathrm{k}_{\mathrm{n}}$ and $\mathrm{k}_{\mathrm{tx}}$. The values thus obtained are then normalized by the length of contact of the three point damper and multiplied by L, the length of contact of the cylindrical damper. The stiffness for each contact point can then be determined by dividing the obtained values by the number of the chosen contact points.

\section{Funding sources}

The Octopus test rig used in this paper has been developed within the "PRIN" (National Interest Research Project) funded by MIUR (Italian Ministry of Education, Universities and Research). The Damper test rig used in this paper has been developed using funds by the AERMEC Lab.

\section{Competing interests}

Chiara Gastaldi declares that she has no conflict of interest. Emanuele Grossi declares that he has no conflict of interest. Teresa M. Berruti declares that she has no conflict of interest.

\section{References}

Allara M., Firrone C. M., and Gola M. M. (2008). A contact model for nonlinear forced response prediction of turbine blades: Calculation techniques and experimental comparison. ASME Turbo Expo 2008: Power for Land, Sea, and Air. 5: 573-582. https://doi.org/10.1115/GT2008-51231. 
Allara M. (2009). A model for the characterization of friction contacts in turbine blades. Journal of Sound and Vibration. 320 (3): 527-544. https://doi.org/10.1016/j.jsv.2008.08.016.

Berruti T., Firrone C. M., Pizzolante M., and Gola M. M. (2007). Fatigue damage prevention on turbine blades: Study of underplatform damper shape. Key Engineering Materials. 347: 159-164. https://doi.org/10.4028/www.scientific.net/ KEM.347.159.

Berruti T., Firrone C. M., and Gola M. M. (2011). A test rig for noncontact traveling wave excitation of a bladed disk with underplatform dampers. Journal of Engineering for Gas Turbines and Power. 133 (3): 032502. https://doi.org/10.1115/ 1.4002100

Berruti T. and Maschio V. (2012). Experimental investigation on the forced response of a dummy counter-rotating turbine stage with friction damping. Journal of Engineering for Gas Turbines and Power. 134 (12): 122502. https://doi.org/10.1115/ 1.4007325 .

Botto D., Lavella M., and Gola M. M. (2012). Measurement of contact parameters of flat on flat contact surfaces at high temperature. ASME Turbo Expo 2012: Turbine Technical Conference and Exposition. 7: 1325-1332. https://doi.org/ 10.1115/GT2012-69677.

Brändlein J., Eschmann P., Hasbargen L., and Weigand K. (1999). Ball and Roller Bearings: Theory, Design and Application, 3rd Edition. John Wiley and Sons. ISBN: 978-0-471-98452-8.

Castanier M. P. and Pierre C. (2006). Modeling and analysis of mistuned bladed disk vibration: Status and emerging directions. Journal of Propulsion and Power. 22 (2): 384-396. https://doi.org/10.2514/1.16345.

Charleux D., Gibert C., Thouverez F., and Dupeux J. (2006). Numerical and experimental study of friction damping in blade attachments of rotating bladed disks. International Journal of Rotating Machinery. 2006: 1-13. https://doi.org/ 10.1155/IJRM/2006/71302.

Cigeroglu E., An N., and Menq C. H. (2007). Wedge damper modeling and forced response prediction of frictionally constrained blades. ASME Turbo Expo 2007: Power for Land, Sea, and Air. 5: 519-528. https://doi.org/10.1115/GT20072796.

Cigeroglu E., An N., and Menq C. H. (2009). Forced response prediction of constrained and unconstrained structures coupled through frictional contacts. ASME Journal of Engineering for Gas Turbines and Power. 131 (2): 022505. https:// doi.org/10.1115/1.2940356.

Craig R. R. and Bampton M. C. C. (1968). Coupling of substructures for dynamic analysis. AIAA Journal. 6 (7): 13131319. https://doi.org/10.2514/3.4741.

Firrone C. M. and Berruti T. (2012). Non contact measurement system with electromagnets for vibration tests on bladed disks. In: Applied Measurement Systems, edited by Zahurul Haq. InTech. 77-108. https://doi.org/10.5772/36948.

Firrone C. M., Zucca S., and Gola M. M. (2009). Effect of static/dynamic coupling on the forced response of turbine bladed disks with underplatform dampers. ASME Turbo Expo 2009: Power for Land, Sea, and Air. 6: 429-440. https://doi.org/ 10.1115/GT2009-59905.

Gastaldi C. and Gola M. M. (2016a). On the relevance of a microslip contact model for under-platform dampers. International Journal of Mechanical Sciences. 115-116: 145-156. https://doi.org/10.1016/j.ijmecsci.2016.06.015.

Gastaldi C. and Gola M. M. (2016b). Pre-optimization of asymmetrical underplatform dampers. Journal of Engineering for Gas Turbines and Power. 139 (1): 012504. https://doi.org/10.1115/1.403419.

Gola M. M. and Liu T. (2014). A direct experimental-numerical method for investigations of a laboratory under-platform damper behavior. International Journal of Solids and Structures. 51 (25-26): 4245-4259. https://doi.org/10.1016/j. ijsolstr.2014.08.011.

Harris T. A. and Kotzalas M. N. (2006). Rolling Bearing Analysis, 5th Edition. CRC Press. ISBN: 0849381673.

Hills D. A., Nowell D., and Sackfield A. (1993). Mechanics of Elastic Contacts Oxford: Butterworth-Heinemann. ISBN: 978-0-7506-0540-3.

Lavella M. (2016). Contact properties and wear behaviour of nickel based superalloy René 80. Metals. 6 (7): 159. https://doi. org/10.3390/met6070159.

Lavella M., Botto D., and Gola M. M. (2011). Test rig for wear and contact parameters extraction for flat-on-flat contact surfaces. ASME/STLE 2011 International Joint Tribology Conference. 307-309. https://doi.org/10.1115/IJTC2011-61234.

Panning L., Popp K., Sextro W., Goetting F., Kayser A., and Wolter I. (2004). Asymmetrical underplatform dampers in gas turbine bladings: Theory and application. ASME Turbo Expo 2004: Power for Land, Sea, and Air. 6: 269-280. https://doi. org/10.1115/GT2004-53316.

Petrov E. P. (2004). A method for use of cyclic symmetry properties in analysis of nonlinear multiharmonic vibrations of bladed discs. ASME Journal of Turbomachinery. 126 (1): 175-183. https://doi.org/10.1115/1.1644558.

Petrov E. P. (2007). Direct parametric analysis of resonance regimes for nonlinear vibrations of bladed discs. ASME Journal of Turbomachinery. 129 (3): 495-502. https://doi.org/10.1115/1.2720487. 
Petrov E. P. (2012). Analysis of flutter-induced limit cycle oscillations in gas-turbine structures with friction, gap and other nonlinear contact interfaces. Journal of Turbomachinery. 134 (6): 061018. https://doi.org/10.1115/1.4006292.

Petrov E. P. and Ewins D. J. (2007). Advanced modelling of underplatform friction dampers for analysis of bladed disk vibration. ASME Journal of Turbomachinery. 129 (1): 143-150. https://doi.org/10.1115/1.2372775.

Siewert C., Panning L., Wallaschek J., and Richter C. (2009). Multiharmonic forced response analysis of a turbine blading coupled by nonlinear contact forces. ASME Turbo Expo 2009: Power for Land, Sea, and Air. 6: 231-243. https://doi.org/ 10.1115/GT2009-59201. 